\title{
COMUNICAÇÃO
}

\section{IMPACTO ECONÔMICO DO INTERVALO DE PARTOS EM REBANHOS BOVINOS LEITEIROS}

\author{
Economic impact of calving interval on dairy cattle herds
}

\author{
Marcos Aurélio Lopes ${ }^{1}$, Fabiana Alves Demeu², Glauber dos Santos², Milton Ghedini Cardoso
}

\begin{abstract}
RESUMO
Conduziu-se esta pesquisa com o objetivo de avaliar e quantificar o impacto econômico do intervalo de partos em rebanhos de gado de leite. Foram simulados, no software Dimensionamento e Evolução de Rebanhos Bovinos, três rebanhos bovinos leiteiros, de um sistema de produção hipotético composto por 25 fêmeas, da raça holandesa, com média de produção diária de $15 \mathrm{~kg}$, com idade ao primeiro parto de 30 meses, mantidas em 200 ha cultivados com pastagem de Brachiaria decumbens Stapf. com capacidade de suporte de 1UA/ha/ano. A equivalência entre as categorias animais utilizadas foram: matrizes 1,30; fêmeas de 0 - 1 ano 0,30 ; fêmea de 1-2 anos 0,71; fêmeas de 2-3 anos 1,01 UA/ha. Os três rebanhos possuiam as mesmas taxas de descarte (20\%) e mortalidade (3\% de 0 a 1 ano; $2 \%$ de a 1 a 2 anos e $1 \%$ de 2 a 3 anos) diferindo apenas as taxas de natalidade, quais sejam 100; 80 e $60 \%$, para os casos $1 ; 2$ e 3, respectivamente refletindo em intervalos de partos de 365, 510 e 657 dias. A simulação do impacto econômico foi realizada no aplicativo MS Excel®. O intervalo de partos, afetou diretamente a composição e a evolução do rebanho e influenciou a rentabilidade da atividade leiteira, sendo mais eficiente o índice de natalidade $100 \%$, ou seja, intervalo de partos de 12 meses.
\end{abstract}

Termos para indexação: Bovinocultura de leite, índices zootécnicos, rentabilidade, simulação.

\section{ABSTRACT}

The aim of this study was to quantify and evaluate the economic impact of calving interval on dairy herd. A simulation using a Dimensioning and Evolution of Bovine Herd software was conducted on three dairy herds from a hypothetical production system composed by 25 females of Holstein breed, having an average of $15 \mathrm{~kg}$ daily milk production, at an age of 30 months after the first calving, kept in a 200 ha Brachiaria decumbens Stapf. pasture presenting an 1AU/ha/year support capacity. The equivalence among the tested animal categories were: dam cows 1.30 ; females of $0-1$ year 0.30 ; females of $1-2$ years 0.71 ; females of 2-3 years $1.01 \mathrm{n}$ AU/ ha. The three herds showed the same culling rates (20\%) and mortality rates (3\% for 0 to 1 year; $2 \%$ for 1 to 2 years and $1 \%$ for 2 to 3 years), only the birth rates differed, namely $100 ; 80$ and $60 \%$ for the cases $1 ; 2$ and 3, respectively as reflecting in calving intervals of 365, 510 and 657 days. The simulation of the economic impact was done by the MS Excel(r) applicative. Calving interval not only directly affected the herd composition and evolution but also influenced dairy business profitability, the most efficient birth rate was that of $100 \%$, in other words, calving interval of 12 months.

Index terms: Diary cattle production, performance indices, profitability, simulation.

\section{(Recebido em 15 de outubro de 2007 e aprovado em 14 de abril de 2008)}

A utilização de índices para avaliar a eficiência na pecuária leiteira tem sido uma prática constante. Visando a auxiliar pecuaristas e técnicos nessa tarefa, Lopes et al. (2004, 2005) calcularam diversos índices técnicos e gerenciais. No entanto, alguns índices zootécnicos tais como: idade ao primeiro parto, taxa de natalidade, taxa de descarte e taxa de mortalidade possuem significativa importância, pois indicam produtividade, desempenho e evolução de rebanhos, assim como, rentabilidade de sistemas de produção de leite.
A lucratividade da atividade pecuária pode ser avaliada pelos índices zootécnicos, uma vez que eles estão relacionados à produção e, conseqüentemente, aos lucros do produtor. Assim, produtores e técnicos devem estar atentos para calcular os índices zootécnicos e identificar aqueles que apresentam maior desvio em relação a uma situação desejável e afetando a rentabilidade da atividade, identificando os pontos de estrangulamento, maximizando a produção e minimizando os custos.

\footnotetext{
'Doutor em Zootecnia, Professor - Departamento de Medicina Veterinária/DMV - Universidade Federal de Lavras/UFLA - Cx. P. 3037 - 37200-000 Lavras, MG - malopes@dmv.ufla.br - Bolsista CNPq

ZZootecnistas - Departamento de Medicina Veterinária/DMV - Universidade Federal de Lavras/UFLA - Cx. P. 3037 - $37200-000$ - Lavras, MG fabianademeu@bol.com.br; glauber_zoo@yahoo.com.br - Bolsistas da CAPES

${ }^{3}$ Médico Veterinário - Departamento de Medicina Veterinária/DMV - Universidade Federal de Lavras/UFLA - Cx. P. 3037 - $37200-000$ - Lavras, MG cardoso_vet@yahoo.com.br
} 
Segundo Lemos et al. (1992) um índice indicativo da eficiência reprodutiva é a idade ao primeiro parto, indicativo de precocidade sexual e, portanto, de elevada importância econômica, uma vez que marca o início da vida produtiva de uma fêmea leiteira e influencia os custos de reposição das matrizes. Lozano et al. (2002) afirmaram que o anestro após o parto está ligado a nutrição, uma vez que a fertilidade dos bovinos está diretamente ligada a esta variável. Santos et al. (2001) relataram que a redução do intervalo de partos está relacionada ao nascimento de mais bezerras e existindo a possibilidade de uma seleção mais aprimorada e um maior número de novilhas para descarte.

Em estudos realizados com rebanhos mestiços holandês x zebu, Araújo Neto et al. (2002) observaram um intervalo de partos de 14,27 meses; Marques et al. (2002) relatam média de 19 meses e Facó et al. (2002) 407,51 dias, aproximadamente 13,56 meses, semelhante as observações de Freitas et al. (2002), que descrevem uma média de 412,72 dias $(13,75)$ meses.

O intervalo de partos reflete diretamente nos custos da produção. Yamaguchi et al. (1997) trabalhando com vacas holandesas, em confinamento salientaram que uma vaca seca por período de 60 dias representa um custo médio em torno de US\$206,00, equivalente a 665 litros de leite. A produtividade do rebanho está relacionada ao intervalo de partos como relatado por Beretta et al. (2001), ao afirmarem que esse índice afetou diretamente a proporção de novilhas de reposição em estoque, sendo o valor dependente da taxa de natalidade. A medida que o intervalo de partos se estende, diminui a taxa de natalidade, ocorre menor nascimento de bezerros e, com isso, uma das fontes de receita da propriedade leiteira, que é a venda de bezerros (as) e novilhas, tende a declinar.

Cardoso \& Lopes (2006) após analisarem a influência de diferentes índices zootécnicos na composição e evolução de rebanhos leiteiros ao longo dos anos, concluiram que a taxa de natalidade tem a maior influência na evolução, seguida pela idade ao primeiro parto, taxa de descarte e taxa de mortalidade. Considerando a importância do tema da influência dos índices zootécnicos na rentabilidade, nesta pesquisa, objetivou-se avaliar e quantificar o impacto econômico do intervalo de partos em rebanhos de gado de leite.

Esta pesquisa foi desenvolvida no Laboratório de Informática Aplicada à Medicina Veterinária, na Universidade Federal de Lavras - MG. Foram simulados três rebanhos bovinos leiteiros com diferentes intervalos de partos, de um sistema de produção hipotético composto por 25 fêmeas, da raça holandesa, com idade ao primeiro parto de 30 meses, mantidas em 200 ha cultivados com pastagem de Brachiaria decumbens Stapf. com capacidade de suporte de 1UA/ha/ano. Cada um dos rebanhos foi cadastrado no software Sistema Computacional para Dimensionamento e Evolução de Rebanhos Bovinos (LOPES, 2000), o qual estimou o dimensionamento e a evolução ao longo dos anos. Tal software utilizou os valores de equivalência de categorias animais proposto por Lopes et al. (2000), ou seja: matrizes 1,30; fêmea de 0 a 1 ano, 0,30; fêmea de 1 a 2 anos, 0,71; fêmea de 2 a 3 anos, 1,01 UA/ha. Nos três casos foi adotada a inseminação artificial, não sendo, portanto, utilizado reprodutores.

A taxa de descarte, calculada aqui como o descarte involuntário mais o voluntário, foi de $20 \%$ (CARDOSO \& NOGUEIRA, 2001; SANTOS et al., 2001). A mortalidade foi de 3; 2 e $1 \%$ nas categorias animais de 0 a 1 ano; 1 a 2 anos; e 2 a 3 anos, respectivamente, com base nas pesquisas de Araújo Neto et al. (2002) e Campos \& Ferreira (1996). Os três rebanhos tinham as mesmas taxas de descarte e mortalidade, diferindo apenas as taxas de natalidade, quais sejam 100; 80 e 60\%, para os casos 1; 2 e 3, respectivamente, refletindo em intervalos de partos de 365 , a maior eficiência no rebanho; 510 dias, que é um pouco acima dos dados obtidos por Araújo Neto et al. (2002) e 657 dias que é próximo às observações de Marques et al. (2002). Com estes índices, os rebanhos estabilizariam com 14; 19 e 31 anos, considerando que apenas os animais nascidos na fazenda seriam utilizados nesta evolução até completar a estabilização do rebanho; ou seja, não haveria a compra de animais.

O investimento inicial com a aquisição das 25 vacas, para as três simulações, foi de $\mathrm{R} \$ 67.000,00$ (25 x R \$2.680,00), enquanto que a infraestrutura (máquinas, equipamentos, implementos e benfeitorias) foi de $\mathrm{R} \$ 79.221,71$, de acordo com o trabalho de Lopes et al. (2007). Foi considerado para o inventário do rebanho, a composição ideal de acordo com a capacidade de suporte da propriedade, multiplicado pelo valor de venda de cada animal, de acordo com a sua categoria. Definiu-se como preço de venda o custo operacional efetivo (valor referente às despesas que ocorreram efetivo desembolso, tais como: alimentação, mão-de-obra, medicamentos etc) para formação de cada animal, acrescido de uma margem de ganho de $10 \%$.

As receitas foram auferidas com a venda do leite, considerando uma produção média de $15 \mathrm{~kg}$ por vaca em lactação, durante um período de 305 dias, e com a venda de machos ao nascer ( $\mathrm{R} \$ 50,00)$. Para comercialização das vacas, utilizou-se média ponderada, onde $80 \%$ das vacas de descarte foram comercializadas a $\mathrm{R} \$ 2.680,00$, por se tratar de descarte voluntário, e $20 \%$, devido a problemas no aparelho mamário e nos membros locomotores (descarte 
involuntário), gerando uma receita média de $\mathrm{R} \$ 882,00 /$ vaca, vendidas para o abate, considerando o preço de arroba de vaca gorda de $\mathrm{R} \$ 49,00$, com peso médio de 18 arrobas.

O custo anual dos animais foi definido de acordo com a categoria e o preço dos insumos do comércio local de Lavras. O custo operacional efetivo diário das vacas "solteiras" (vacas que pariram e que não foram inseminadas e/ou foram inseminadas e apresentaram prenhes negativa, possuindo grande período de serviço, com conseqüente intervalo de parto alto) foi de $\mathrm{R} \$ 2,89$ (custos fixos e variáveis diários de cada animal), o que correspondeu a um valor de $\mathrm{R} \$ 213,40$; $\mathrm{R} \$ 445,10$; e $\mathrm{R} \$ 1.011,15$ para os períodos secos com duração de 60, 205 e 352 dias, respectivamente. Para as fases de cria e recria, optou-se por desenvolver uma planilha especificamente para este fim, considerando todos os manejos: sanitário, zootécnico e alimentar necessários para que as fêmeas atingissem uma idade de 30 meses para o primeiro parto. Nos três casos em questão, as fêmeas de 0 a 1 ano tiveram um custo operacional de $\mathrm{R} \$ 640,00$; de 0 a 2 anos, $\mathrm{R} \$ 1.500,00$; e de 0 a 3 anos, $R \$ 2.680,00$, sendo este mesmo valor considerado como custo para uma vaca em lactação. Para calcular a margem líquida do leite, adotou-se para a venda do leite o valor de $\mathrm{R} \$ 0,49$ e o custo operacional total do leite virtual de R\$0,32 (LOPES et al., 2007). O termo "leite virtual", criado por Lopes \& Lopes (1999), significa a quantidade de leite, em kg, resultante da conversão dos valores apurados com as vendas de animais. Esse valor pode ser tomado como referência para o produtor avaliar se a atividade leiteira como um todo e, principalmente a cria e recria de animais, está sendo viável economicamente.

Para realizar a análise econômica optou-se por trabalhar com as seguintes variáveis:

- Margem $(\mathrm{M})=$ venda de animais $(\mathrm{V})$ mais a margem líquida do leite (MLL) menos o investimento realizado em animais (IA), correspondendo a $\mathrm{M}=(\mathrm{V}+\mathrm{MLL})-\mathrm{IA}$.

- Receita de ganho da atividade leiteira (RL)=Venda de animais (VL) + margem líquida do leite MLL).

- Inventário (I) = valor dos investimentos da propriedade em animais e infraestrutura, sendo que, no caso dos animais, foi considerado o valor do custo operacional de formação de cada animal, acrescido de uma margem de ganho de $10 \%$.

- Acumulado (A)=receita da venda de animais (VA) mais a margem líquida do leite (MLL) do ano da atividade menos os valores residuais dos anos anteriores $\left(\mathrm{M}_{\text {ant }}\right)$ somados ao desembolso do ano em questão $\left(\mathrm{M}_{\text {ano }}\right)$ até atingir a estabilização $\left.(A=V A+M L L)-M_{\text {anot }} M_{\text {ant }}\right)$.

- Margem líquida acumulada do leite (MLacum)=produção de leite durante a lactação (PLT) vezes o valor da venda do leite (VVL) menos o custo operacional total do leite virtual (COTL) vezes a quantidade de vacas em lactação (VL) MLacum=PLT*(VVLCOTL)*VL).

- A porcentagem do investimento pago no ano de estabilização (PIPAE) foi calculado considerando a margem líquida acumulada do leite mais a venda de animais dividida pela margem acumulada até o ano de estabilização de cada propriedade $(\mathrm{PIPAE}=(\mathrm{MLL}+\mathrm{VA}) / \mathrm{Macum})$.

$\mathrm{Na}$ simulação realizada é possível verificar que quanto maior é o período para estabilizar o rebanho, maiores são os custos no ano da estabilização (Tabela 1). Os animais improdutivos são os grandes responsáveis pelo aumento de custos da propriedade, com destaque para as vacas "solteiras", uma vez que as novilhas são animais essenciais para reposição e melhoramento do rebanho. Vacas "solteiras" por períodos prolongados são animais que estão no rebanho gerando custos operacionais efetivos diários de $\mathrm{R} \$ 2,89 /$ vaca, referentes a despesas com alimentação, mão-de-obra, medicamentos etc. Transcorrido o período seco, com uma duração ideal de 60 dias, elas deveriam estar paridas, gerando receitas na ordem de $\mathrm{R} \$ 7,35 /$ dia, considerando uma produtividade média diária de $15 \mathrm{~kg}$ e o preço de venda de $\mathrm{R} \$ 0,49 / \mathrm{kg}$. No entanto, quando se observa maiores intervalos de parto, os animais problema, além de não gerarem receita, têm seus custos aumentados. Como é de se presumir, a medida que se aumentou o intervalo de partos, aumentou o número de vacas "solteiras", $(00,19$ e 44) na proporção de $0 \% ; 17,12 \%$; e 38,26\% em relação ao total de matrizes, respectivamente, para rebanhos 1,2 e 3 , cuja estabilização ocorreu nos 14,19 e 31 anos, respectivamente (Tabela 2 ). O número de animais improdutivos é menor quando a estabilização ocorreu aos 14 anos, apresentando 107 animais (50,47\%), 112 $(54,90 \%)$ e $129(64,50 \%)$, respectivamente, para os rebanhos 1, 2 e 3. Os animais que estão compondo o rebanho 1 apresentam menor peso em relação aos outros anos analisados; portanto, é necessário um maior número de animais para compor a capacidade de suporte. Assim, as diferentes categorias de novilhas apresentam menor UA/ha em relação às vacas "solteiras" (Tabela 2); uma vez que o dimensionamento do rebanho é calculado em função da capacidade de suporte, será observada maior quantidade de animais e aproximadamente o mesmo número de $\mathrm{UA} / \mathrm{ha}$, visto que a composição dos rebanhos ficou muito próximas do ideal que seria $200 \mathrm{UA} / \mathrm{ha}$, sendo 200,4; 200,2 e 199,9 UA/ha, para a estabilização nos anos 14 (rebanho 1), 19 (rebanho 2) e 31 (rebanho 3), respectivamente. 
No rebanho 2, houve estabilização no ano 19, se comparado aos rebanhos 1 (ano de estabilização 14) e rebanho 3 (ano de estabilização 31), verifica-se que no rebanho 1 não há vacas "solteiras", pois todas foram fecundadas dentro do período voluntário de espera e, no segundo caso (ano 19), observa-se ocorrência de 24 vacas "solteiras", ou seja, $11,76 \%$ do rebanho, e as quantidades das diferentes categorias de novilhas são inferiores. $\mathrm{Na}$ comparação dos dados entre os casos 2 e 3 (19 e 31 anos), o resultado é mais semelhante, sendo mais expressiva a quantidade de vacas "solteiras" $(44 ; 38,26 \%)$ no ano de estabilização 31 e gradativamente se observa um menor número de novilhas para reposição, o que poderá afetar o melhoramento genético do rebanho, além de diminuir as receitas auferidas com a venda de animais. Resultados semelhantes são apontados por Beretta et al. (2001), quando constataram que a baixa taxa de natalidade afetou a composição do rebanho, havendo uma grande quantidade de vacas que precisam continuar no rebanho por falta de novilhas para reposição.

Com relação à receita bruta da propriedade no ano de estabilização (Tabela 1), observa-se que esta é maior quando a estabilização do rebanho ocorre em menor tempo $\mathrm{R} \$ 313.288,25$, aos 14 anos; $\mathrm{R} \$ 256.358,25$, aos 19 anos e $\mathrm{R} \$ 198.252,25$ com a estabilização aos 31 anos de atividade. Tal fato pode ser explicado por haver um maior número de
Tabela 1 - Resumo econômico, em R \$, de três rebanhos leiteiros, de acordo com os anos de estabilização.

\begin{tabular}{lccc}
\hline & \multicolumn{3}{c}{ Rebanhos } \\
\cline { 2 - 3 } & $01 *$ & $02^{* *}$ & $03 * * *$ \\
\hline $\begin{array}{l}\text { Margem de } \\
\text { ganho no ano de } \\
\text { estabilização }\end{array}$ & $14.276,25$ & $(9.936,15)$ & $(63.835,80)$ \\
$\begin{array}{l}\text { Receita bruta no } \\
\text { ano da }\end{array}$ & & & \\
estabilização & $313.288,25$ & $256.358,25$ & $198.252,25$ \\
$\begin{array}{l}\text { Receita da } \\
\text { venda de }\end{array}$ & $46.520,00$ & $43.392,00$ & $39.088,00$ \\
animais & $(15 \%)$ & $(17 \%)$ & $(20 \%)$ \\
$\begin{array}{l}\text { Receita da } \\
\text { venda de leite }\end{array}$ & $\begin{array}{c} \\
(85 \%)\end{array}$ & $\begin{array}{c}212.966,25 \\
(83 \%)\end{array}$ & $\begin{array}{c}159.164,25 \\
(80 \%)\end{array}$ \\
\hline
\end{tabular}

* estabilização ocorrida aos 14 anos; ** estabilização ocorrida aos 19 anos; *** estabilização ocorrida aos 31 anos

vacas em lactação (105) no primeiro caso, enquanto decresce na estabilização nos anos 19 e 31, com 92 e 71 vacas, respectivamente (Tabela 2 ).

Os dados demonstram que a percentagem da venda do leite é maior à medida que a estabilização do rebanho ocorre em menor tempo, representando 85,83 e $80 \%$ da receita (Tabela 1), para os casos 1,2 e 3 , respectivamente. À medida que o intervalo de partos proporcionou uma

Tabela 2 - Composição dos rebanhos no ano da estabilização e suas respectivas lotações.

\begin{tabular}{|c|c|c|c|c|c|c|c|c|c|}
\hline \multirow{3}{*}{ Categoria animal } & \multicolumn{9}{|c|}{ Rebanhos } \\
\hline & \multicolumn{3}{|c|}{$01 *$} & \multicolumn{3}{|c|}{$02 * *$} & \multicolumn{3}{|c|}{$03 * * *$} \\
\hline & CI & $\mathrm{L}$ & $\mathrm{CAE}$ & $\mathrm{CI}$ & $\mathrm{L}$ & CAE & $\mathrm{CI}$ & $\mathrm{L}$ & CAE \\
\hline Vacas em lactação & 105 & 136,5 & 119 & 92 & 119,6 & 95 & 71 & 92,3 & 71 \\
\hline Descarte de vacas em lactação & 0 & 0 & 20 & 0 & 0 & 17 & 0 & 0 & 13 \\
\hline Vacas solteiras & 0 & 0 & 0 & 19 & 24,7 & 24 & 44 & 57,2 & 47 \\
\hline Descarte de vacas solteiras & 0 & 0 & 0 & 0 & 0 & 4 & 0 & 0 & 9 \\
\hline Fêmeas de $0-1$ anos & 44 & 13,2 & 60 & 38 & 11,4 & 48 & 36 & 10,8 & 36 \\
\hline Descarte de fêmeas 0 - 1 anos & 0 & 0 & 0 & 0 & 0 & 0 & 0 & 0 & 0 \\
\hline Fêmeas de 1 - 2 anos & 43 & 30,5 & 49 & 37 & 26,2 & 42 & 33 & 23,4 & 32 \\
\hline Descarte de fêmeas de $1-2$ anos & 0 & 0 & 0 & 0 & 0 & 0 & 0 & 0 & 0 \\
\hline Fêmeas de 2 - 3 anos & 20 & 20,2 & 20 & 18 & 18,18 & 18 & 16 & 16,16 & 15 \\
\hline Descarte de fêmeas de $2-3$ anos & 0 & 0 & 0 & 0 & 0 & 0 & 0 & 0 & 0 \\
\hline Total de animais & 212 & 200,4 & 268 & 204 & 200,2 & 248 & 200 & 199,9 & 223 \\
\hline Animais improdutivos & 107 & 63,9 & 129 & 112 & 80,5 & 136 & 129 & 107,5 & 139 \\
\hline Percentual de animais improdutivos & 50,4 & & & 54,9 & & & 64,5 & & \\
\hline
\end{tabular}


estabilização mais rápida, a proporção de vacas em lactação foi maior quando comparada às outras situações, aumentando a produção de leite. Assim se obtém maior receita e menor custo, uma vez que as vacas em lactação são as responsáveis pela geração da receita na propriedade. Almeida Júnior et al. (2002), estudando o efeito da venda de animais na rentabilidade de um sistema de produção de leite, encontraram valores médios de 16,95\%; enquanto que Lopes et al. (2004, 2005, 2006) constataram que a venda de animais representou $10,16 \%$, $12,40 \%$ e $12,41 \%$ da receita da atividade leiteira, respectivamente.

Na Tabela 3 está o resumo econômico dos três rebanhos ao longo dos anos até atingir a estabilização. As margens que se mostraram negativas nos três casos, também aumentam à medida que o tempo de estabilização se prolonga, mostrando-se menor no ano 14 ( $\mathrm{R} \$ 143.931,50)$, 19 (R\$262.179,65) e, por fim, 31 (R \$955.930,20) (Tabela 3), pois quanto maior o tempo para estabilização maiores serão os custos para manutenção desse rebanho. Tal fato denota que os valores apurados com a renda de leite e de animais de descarte não foi suficiente para cobrir as depesas da atividade leiteira, havendo, portanto, necessidade de injeção de recursos de outras fontes. Outro fator que contribuiu para esse aumento foi o fato de haver um maior número de animais improdutivos no rebanho (107, 112 e 129) (Tabela 2), a medida que aumentaram os anos de estabilização, sendo então maiores as despesas geradas com menor geração de receita, como relatado por Beretta et al. (2001).

É importante observar que os empreendimentos exigem um investimento inicial e a busca da recuperação do capital ao longo dos anos da atividade. Assim, quanto maior a eficiência, espera-se maior lucratividade e menor será o tempo de recuperação do investimento. Na simulação realizada, esse fator não foi desprezado e buscou-se estimar o impacto do intervalo de partos sobre o retorno do investimento, em função do ano de estabilização. Foi possível verificar que houve diferença, pois no rebanho 1 ocorreu $86 \%$ do retorno do investimento preservando o patrimônio da propriedade, enquanto que nos rebanhos 2 e 3 houve retorno de somente 78 e $58 \%$, respectivamente. Tais taxas são bastante baixas e são decorrentes, principalmente de dois fatores. $\mathrm{O}$ primeiro diz respeito ao tamanho do rebanho inicial, composto por 25 vacas, que correspondia a 32,5 UA, sendo bastante pequeno e realmente necessitando de muito mais tempo para evoluir e estabilizar, mesmo na situação com intervalo de parto ideal (12 meses, equivalente a uma taxa de natalidade de $100 \%$ ). O segundo fator é a produtividade de leite por ha/ano no ano da estabilização de 2.401,88; 2.104,50 e 1.624,13 1/ha/ano, para os rebanhos estabilizados com 14, 19 e 31 anos, respectivamente. Esses valores são bastante baixos quando comparados aos $19.710 \mathrm{l} / \mathrm{ha} / \mathrm{ano}$ obtidos por Rassini et al. (2003). A situação é ainda pior quando se considera a produtividade média de leite do ano zero até o ano da estabilização $(1.125,45 ; 913,86$ e 717,70 1/ ha/ano), respectivamente.

$\mathrm{O}$ intervalo de partos, afetando diretamente a composição e a evolução do rebanho, influenciou a rentabilidade da atividade leiteira, sendo mais eficiente o índice de natalidade $100 \%$, ou seja, intervalo de partos de 12 meses. Com a melhoria deste índice zootécnico se obtém maior eficiência do rebanho, com maior número de vacas em lactação e menor número de vacas "solteiras", aumentando a eficiência reprodutiva e a rentabilidade do sistema de produção.

Tabela 3 - Resumo econômico de três rebanhos leiteiros, ao longo dos anos até atingir a estabilização.

\begin{tabular}{lccc}
\hline \multicolumn{1}{c}{ Especificação } & \multicolumn{3}{c}{ Rebanhos } \\
\cline { 2 - 4 } & 01 & 02 & 03 \\
\hline Custo & $1.049 .500,00$ & $1.295 .823,90$ & $2.284 .645,20$ \\
Margem & $(143.931,50)$ & $(262.179,65)$ & $(955.930,20)$ \\
Somatório das receitas bruta & $1.942 .185,50$ & $2.152 .524,25$ & $2.948 .240,00$ \\
Percentual das receitas sobre os custos & $54 \%$ & $60 \%$ & $77 \%$ \\
Receita da venda de animais & $287.774,00$ & $361.366,00$ & $587.677,25$ \\
Receita da venda de leite & $1.654 .411,50$ & $1.791 .158,25$ & $2.360 .562,75$ \\
\% venda de animais & $15 \%$ & $17 \%$ & $20 \%$ \\
\% venda de leite & $85 \%$ & $83 \%$ & $80 \%$ \\
Valor do inventário do rebanho & $374.740,00$ & $374.620,00$ & $265.736,00$ \\
\hline
\end{tabular}

* estabilização ocorrida aos 14 anos; ** estabilização ocorrida aos 19 anos; *** estabilização ocorrida aos 31 anos 


\section{REFERÊNCIAS BIBLIOGRÁFICAS}

ALMEIDA JÚNIOR, G. A. de; LOPES, M. A.; PINATTO, F. Efeito da venda de animais na rentabilidade de um sistema intensivo de produção de leite tipo B no estado de São Paulo. In: REUNIÃO ANUAL DA SOCIEDADE BRASILEIRA DE ZOOTECNIA, 39., 2002, Recife. Anais... Recife, 2002. CD-ROM.

ARAÚJO NETO, R. B. de; MAGALHÃES, J. A.; LEAL, J. A.; NASCIMENTO, M. P. S. B. C.; NASCIMENTO, H. T. S.; LOPES, E. A.; RODRIGUES, B. H. N.; LEAL, T. M.; ITALIANO, E. C.; LIMA, V. M. B. Coeficientes técnicos. Brasília, DF: Embrapa, 2002. Disponível em: <http:// isistemasdeproducao.cnptia.embrapa.br/FontesHTMLt' Leite/LeiteMeioNorte/coeficientes.html?. Acesso em: 19 jul. 2007.

BERETTA, V.; LOBATO, F. P.; MIELITZ, N.

Produtividade e eficiência biológica de sistemas pecuários de cria diferindo na idade das novilhas ao primeiro parto e na taxa de natalidade do rebanho no rio grande de sul. Revista da Sociedade Brasileira de Zootecnia, Viçosa, v. 30, n. 4, p. 1278-1286, 2001.

CAMPOS, A. L. de T.; FERREIRA, A. de M. Composição no rebanho e sua importância no manejo. 2. ed. rev. e atual. Brasília, DF: Embrapa, 2006. Disponível em: 〈http:/ 'www.cnpgl.embrapa.br/pastprod/32Instrucao.pdf >1. Acesso em: 18 jul. 2007.

CARDOSO, M. G.; LOPES, M. A. Influência de diferentes índices zootécnicos na composição e evolução de rebanhos bovinos leiteiros. In: CONGRESSO DE INICIAÇÃO CIENTÍFICA DA UFLA CIUFLA, 19., 2006, Lavras. Anais... Lavras: UFLA, 2006. p. 335.

CARDOSO, V. L.; NOGUEIRA, J. R. Efeito da mudança do nível de produção, preço do leite e custos de produção, sobre estratégias ótimas de reposição em sistema de produção de bovinos da raça holandesa. In: REUNIÃO ANUAL DA SOCIEDADE BRASILEIRA DE ZOOTECNIA, 38., 2001, Piracicaba. Anais... Piracicaba: ESALQ, 2001.

FACÓ, O.; MARTINS FILHO, R.; LÔBO, R. N. B. Análise do desempenho reprodutivo de cinco grupos genéticos holandês x gir no Brasil. In: REUNIÃO ANUAL DA SOCIEDADE BRASILEIRA DE ZOOTECNIA, 49., 2002, Recife. Anais... Recife: UFPE, 2002.
FREITAS, M. S.; CAVALCANTI, H.; COSTA, C. N.; FREITAS, A. F.; TORRES, R. A.; RENNÓ, F. P.; ARAÚLO, C. V. Idade ao primeiro parto, intervalo de partos, produção na primeira lactação e produção por dia de intervalo de partos de vacas girolando. In: REUNIÃO ANUAL DA SOCIEDADE BRASILEIRA DE ZOOTECNIA, 49., 2002, Recife. Anais... Recife: UFPE, 2002 .

LEMOS, A. M.; MADALENA, F. E.; TEODORO, R. L.; BARBOSA, R. T.; MONTEIRO, J. B. N. Comparative performance of six Holstein-Friesian x Guzera grades in Brazil 5: age at first calving. Revista Brasileira de Genética, Ribeirão Preto, v. 15, n. 1, p. 73-83, mar. 1992.

LOPES, M. A. Sistema computacional para dimensionar rebanhos bovinos utilizando valores ajustados de equivalência das categorias animais. 2000. 116 p. Tese (Doutorado em Zootecnia) - Universidade Estadual de São Paulo, Jaboticabal, 2000.

LOPES, M. A.; CARDOSO, M. G.; CARVALHO, F. de M.; LIMA, A. L. R.; DIAS, A. S.; CARMO, E. A. do. Efeito do tipo de sistema de criação nos resultados econômicos de sistema de produção de leite na região de Lavras (MG) em 2004 e 2005. Ciência Animal Brasileira, v. 8, n. 3, p. 359-371, 2007.

LOPES, M. A.; LIMA, A. L. R.; CARVALHO, F. de M.; REIS, R. P.; SANTOS, I. C.; SARAIVA, F. H. Efeito do tipo de sistema de criação nos resultados econômicos de sistemas de produção de leite na região de Lavras (MG).

Ciência e Agrotecnologia, Lavras, v. 28, n. 5, p. 1177 1189, 2004.

LOPES, M. A.; LIMA, A. L. R.; CARVALHO, F. de M.; REIS, R. P.; SANTOS, I. C.; SARAIVA, F. H.

Resultados econômicos de sistemas de produção de leite com diferentes níveis tecnólogicos na região de Lavras (MG). Arquivo Brasileiro de Medicina

Veterinária e Zootecnia, Belo Horizonte, v. 57, n. 4, p. 485-493, 2005.

LOPES, M. A.; LIMA, A. L. R.; CARVALHO, F. de M.; REIS, R. P.; SANTOS, I. C.; SARAIVA, F. H. Efeito da escala de produção nos resultados econômicos de sistemas de produção de leite na região de Lavras (MG): um estudo multicasos. Boletim da Indústria Animal, v. 63, n. 3, p. 177-188, 2006. 
LOPES, M. A.; LOPES, D. de C. F. Desenvolvimento de um sistema computacional para cálculo do custo de produção do leite. Revista Brasileira de Agroinformática, v. 2, n. 1, p. 1-12, 1999.

LOPES, M. A.; VIEIRA, P. de F.; CASTRO NETO, P.; MALHEIROS, E. B. Desenvolvimento de um sistema computacional para dimensionamento e evolução de rebanhos leiteiros. Revista Brasileira de Zootecnia, Viçosa, v. 29, n. 5, p. 1511-1519, set./out. 2000.

LOZANO, D. M.; PAIVA, P. C. A.; BANYS, V. L.; SOUZA, J. C.; SAENZ, E. A. C.; OLIVEIRA, E. R. Índices reprodutivos de vacas primíparas red-angus x zebu suplementadas no pós-parto: taxa de prenhêz e concepção ao primeiro serviço. In: REUNIÃO ANUAL DA SOCIEDADE BRASILEIRA DE ZOOTECNIA, 39., 2002, Recife. Anais... Recife: UFPE, 2002. CD-ROM.

MARQUES, V. M.; REIS, R. P.; SÁFADI, T.; REIS, A. J. dos. Custo e escala na pecuária leiteira: estudos de casos em Minas Gerais. Ciência e Agrotecnologia, Lavras, v. 26, n. 5, p. 1027-1034, set./out. 2002.
Disponível em: <http://www.editora.ufla.br/revista/26 5 art19.pdf $>$. Acesso em: 19 jul. 2007.

RASSINI, J. B.; PRIMAVESI, A. C.; PRIMAVESI, O.; TUPY, O.; LÊDO, F. J. S.; FERREIRA, R. P.; BROTEL, M. A.; ALVIM, M. J. Cultivo de alfafa: característica da leguminosa. Brasília, DF: Embrapa, 2003. Disponível em: - shttp://www.cppse.embrapa.br/pesquisa/sistemas-de-1 iproducao/alfafas. Acesso em: 2 set. 2007.

SANTOS, G. T.; CAVALIERI, F. L. B.; MASSUDA, E. M. Alguns aspectos econômicos e de manejo na criação de novilhas leiteiras. Balde Branco, maio 2001. Disponível em: <ihttp://www.nupel.uem.br/novilhas02-2000.pdf > Acesso em: 19 jul. 2007.

YAMAGUCHI, L. C. T.; DURAES, M. C.; COSTA, J. L. da; CARVALHO, L. R. Custos de criação de novilhas até o primeiro parto e manutenção de vacas em sistema confinado, com animais da raça holandesa. In: REUNIÃO ANUAL DA SOCIEDADE BRASILEIRA DE ZOOTECNIA, 1997, Juiz de Fora. Anais... Juiz de Fora: SBZ, 1997. CD-ROM. 